\title{
Breast cancer screening and diagnosis: a glance back and a look forward
}

\author{
Arslaan Javaeed* \\ Department of Pathology, Faculty of Education, University of Ottawa, Ottawa, Canada \\ Received: 12 September 2018 \\ Accepted: 08 October 2018

\section{*Correspondence:} \\ Dr. Arslaan Javaeed, \\ E-mail: ajava102@uottawa.ca \\ Copyright: ( $)$ the author(s), publisher and licensee Medip Academy. This is an open-access article distributed under \\ the terms of the Creative Commons Attribution Non-Commercial License, which permits unrestricted non-commercial \\ use, distribution, and reproduction in any medium, provided the original work is properly cited.
}

\begin{abstract}
The breast cancer has emerged as the commonest malignancy among women and the second most common cancer worldwide after lung cancer. But luckily, the survival rate has also increased significantly to about $98 \%$ in patients with early stage of the disease. It shows that an early and accurate diagnosis will considerably reduce the morbidity and mortality from breast cancer. Therefore, the selection of proper diagnostic technique is of immense importance. Various techniques for screening and diagnosis of breast cancer include conventional methods like self and clinical breast examination, mammography and ultrasound. Magnetic Resonance Imaging (MRI) and nuclear imaging are useful for advanced stages of the disease. Biopsy is the gold standard test for the diagnosis. Present article is an effort review different techniques available for the screening and diagnosis of breast cancer.
\end{abstract}

Keywords: Breast, Cancer, Screening, Diagnosis

\section{INTRODUCTION}

With time, there has been a major change in the lifestyle of people, all around the world. This has led to change in the disease pattern also, shifting from communicable disease to non-communicable diseases. In fact, it can be said that the world is facing dual epidemics, where the western countries are facing rise on non-communicable diseases with decline in the communicable diseases, while the eastern countries are facing reverse scenario. The signs suggest that the future will be dominated by non-communicable diseases, which also includes cancers. There are evidences that breast cancer is strongly associated with un-healthy lifestyles. ${ }^{1}$ the early menarche, increasing obesity, delay in menopause, reduction in parity, decrease in the practice of breastfeeding and use of hormones for various indications are potential risk factors of breast factors that have emerged recently. In this context, the breast cancer has emerged as the commonest malignancy among women and the second most common cancer worldwide after lung cancer. ${ }^{2,3}$ It can also occur in males but on a lesser scale. The ratio of female to male cancer is regarded as $100: 1 .^{4}$ Every year about 1.7 million new cases occur in the world. ${ }^{2}$ There is a wide variation between the incidences of breast cancer, ranging from 19.4 per 100000 people in East Africa to 89.7 per 100000 per people in Western Europe. ${ }^{5}$ Some regions of the world have seen sudden rise on the incidence of breast cancer (as high as 70\%). ${ }^{6}$ In the United States, breast cancer is the second most common cause of female death. ${ }^{4}$ Despite of being a cause of high mortality, this condition has survival rates over a 10 year period in the US, which averages around $85 \%$. This survival rate is significantly increased to about $98 \%$ in patients with early stage of the disease (stages 0 and 1). Even in case of late detection of the breast cancer, effective treatment can improve the quality and length of life. ${ }^{7}$ So it clearly proves that an early diagnosis will significantly reduce the morbidity and mortality from breast cancer. 
Initial techniques for screening and diagnosing breast cancer were limited to mammography and biopsy. The mammography was more of an x-ray of the affected part, than actual mammographic unit, which are used presently. Later on there was an emergence of ultrasonography, which could differentiate cystic mass from solid mass. After a decade from the introduction of ultrasonography, MRI was introduced. Luckily, along with rise in the burden of this disease, there have been improvements in screening and diagnostic techniques. The screening techniques include self-examination, mammography and genetic screening, while the diagnostic techniques include mammography, ultrasound, needle aspiration ad cytology, biopsy and MRI. With increased modalities of diagnosis, the questions of "when to use which technique" is also raised. The researches have shown that different techniques have variable sensitivity and specificity based on the stage of the illness.

Appropriate application of tests is also essential to reduce the saddle of over diagnosis and overtreatment. It poses a burden on health care system as well as puts the patient under economic, physical and mental stress. One such examples of Ductal carcinoma in situ has been described by US Preventive Services Task Force (USPSTF). ${ }^{8}$ After introduction of screening techniques (mainly mammography), the incidence of Ductal carcinoma in situ had increased from 6 per 100000 US Women per year to 37 per 100000 US Women per year. ${ }^{9}$ Because of the less common characteristic of metastasis, its classification as a carcinoma has been under debate and it is recommended to be considered as a risk factor. ${ }^{10}$ In other words, there are is high proportions of "falsepositives", which creates burden on health system and patients.

Current article provides a review of different modalities available for the screening and diagnosis of breast cancer, with their pros and cons.

\section{METHODS}

Present article was drawn from review of about 100 literatures. The goal was to focus on the on recent literature reviews on the topic and also use the significant evidences presented by the articles in the past. It was ensured that the references cited in the current manuscript were relevant to the current scenario and apt to the context. These articles were sourced from indexed medical journals, reputable scholarly articles. Renowned medical websites were also assessed and all of which are well referenced. The keywords used for the literature searches were, "Breast cancer", "Mammography", "Screening", "Diagnosis of Breast Cancer", "MRI", "Nuclear Imaging", "Biopsy" and "Genetic Screening", Care was taken to avoid replication of facts and to stick with only verifiable evidence. This reduced the risk of bias in this review.

\section{RESULTS}

\section{History and examination}

The first form of screening of breast cancer is evidently is the patient's medical history. Breast cancer is common in women above the age of 35 years but it can occur in younger women. Women with a family history of breast cancer have a 3 fold increase in risk of developing breast cancer, themselves. Also women who had early menarche and late menopause are said to be at some-what increased risk. The risk can be attributed to the apparent increased exposure to estrogen in these women, which has strongly been linked to the development of breast cancer. ${ }^{112} \mathrm{~A}$ detailed history containing these factors including age, family history, age at menopause and menarche can lead to identification of women at higher risk of having breast cancer. A thorough history can certainly decrease the excessive burden of unnecessary tests, on the health care service provider as well as on the patients.

For the physical examination, there are basically two types of breast examination; the self breast examination (SBE) and the clinical breast examination (CBE). Both of which are done to detect the presence of a lump in the breast, at very early stage. It should be stated that not all breast lumps are malignant, some are benign, especially those occurring in younger age group which are extremely mobile; the popular fibroadenoma which is referred to as breast mice is an example. Nevertheless, most malignant breast lumps are usually painless, solitary or multiple and they can be fixed, depending on spread. Every breast lump is biopsied to confirm the presence of malignancy. Both SBE and CBE are conducted in the same pattern, with one arm placed to the other side of the head, tensing the pectoralis major muscle upon which the breast lies and the breast is palpated with other arm, along its four quadrants systematically to fill for lumps. CBE is said to have a specificity and sensitivity of $97.11 \%$ and $57.14 \%$ respectively. Compared to that, the sensitivity and specificity of SBE are $87.4 \%$ and $58.3 \%$, respectively. ${ }^{13}$ Although, the numbers suggest that the specificity of CBE is better than SBE, but SBE is the easiest and cheapest method of screening. ${ }^{14}$ The lack of awareness still remains a common barrier for wide spread practice of SBE by the women. Some literatures have shown that there is no improvement in mortality rates after applying $\mathrm{CBE}$, hence making its use generally controversial as it is not diagnostic and yet increases the rate of biopsies. But, still it remains an essential tool for the screening of the said disease. It is generally recommended for women under 40 years and those who do not undergo routine mammography. ${ }^{15,16}$

\section{IMAGING}

\section{Mammography}

For over 30 years, the mammography has remained the mainstay breast cancer screening tool, especially in older 
women. It was considered as one of the most effective mode of screening and diagnosis for breast cancer. It was is widely accepted and regarded as the gold standard for breast cancer screening and it has remarkably improved over the years with new forms and the addition of modernize tools to improve its usage as a screening tool. ${ }^{17}$ In the current article, two forms of mammography are discussed as below, of which the later will fall under recent techniques in breast cancer screening.

Screen-film mammography (SFM): It is regarded as the commonest form of breast cancer imaging. It utilizes $\mathrm{X}$ ray examination of the breast tissue. The breast tissue is usually compressed when it is been done and it is affected by factors such as breast density, age of women, women on hormone replacement therapy, parity, phase of menstrual cycle, family history and body mass index (BMI). It is however said to have a true-positive and false positive rate from $83 \%-95 \%$ to $0.9-6.5 \%$ respectively. A study has showed sensitivity of $68.6 \%$ in women aged 40 to 44 years and $83.3 \%$ in women aged 80 to 89 and specificity values for women not on hormone replacement therapy were $91.4 \%$ and $94.4 \%$ respectively. Sensitivity reduces with breast density, as a study showed sensitivity of $62.9 \%$ and specificity of $89.1 \%$ in women with very dense breast. Nevertheless, when it comes to breast cancer screening, SFM remains the standard that is used but newer technologies still provide a pathway to better screening tools for breast cancer., ${ }^{3,18}$

Full-field digital mammography (FFDM): As technology improved, the use of x-ray films has generally been replaced by digital imaging which has given rise to the FFDM with its many benefits. ${ }^{19}$ FFDM is somewhat similar to SFM because it uses the same imaging technique but allows for real time imaging, but it also allows digital storing of images. However, a study done in the past showed a slight increase in sensitivity up to $95.2 \%$ and specificity up to $41.4 \%$, when compared to that of SFM, which were $91.9 \%$ and $39.3 \%$ respectively. A few setbacks of FFDM, though, include the cost, the amount of digital space required and it a lesser spatial resolution, when compared to film mammography. Just like other screening tests FFDM is affected by factors such as age and breast density. A comparative study done by Pisanno et al showed that FFDM is more accurate for women less than 50 years of age, women with radiographically dense breasts and women in the premenopausal or peri-menopausal period. The study was done with over 40,000 women.

Recent researches have raised questions over the "effectiveness" of mammography, where "effectiveness" has been defined as "ability to reduce mortality resulting from the disease." 20 A randomised trial in Canada, spanning over 25 years, has revealed that no benefit was derived from performing mammography as a screening test. It also disclosed its disadvantage as over-diagnosis of the illness. ${ }^{21}$ Similar findings were obtained in another trial in United Kingdom. ${ }^{17}$ Despite of the surrounding doubts and burden of over-diagnosis, mammography has still remained a corner stone in breast cancer screening techniques in areas with limited resources.

\section{Whole breast ultrasound}

This technique is used more in conjugation with mammography, because it is not very sensitive in itself for screening for breast cancers. It is usually applied in instances were mammography shows abnormal findings. Moreover, in case of dense breast tissue, it may reveal the diagnosis, which could have been hindered by mammography alone. ${ }^{22}$ There are evidences available, which have shown that ultrasound can detect smaller lumps, which may be missed by mammography. For example, in one of the study, the mean size of cancer detected by mammography was $14.7 \mathrm{~mm}$ and by ultrasound, it was $13.5 \mathrm{~mm}^{23}$ The advantages of good tolerance, wide availability, no requirement of intravenous contrast or ionizing radiation and low cost compared to magnetic resonance imaging, make it a good option for a screening/diagnostic tool. However, it has a disadvantage of being greatly operator dependent. Previously there was a limitation of not being able to scan through the entire width of the breast, but recently whole breast ultrasonography has been made available. Nevertheless, its use remains significantly important, which is especially true for the Doppler Ultrasononography. It is able to detect neoangiogenesis which is present in malignancies including breast malignancy. This is supported by a study done by Cosgrove et al, which showed $99 \%$ of malignant lesions containing blood vessels could be revealed by Doppler Ultrasonography. Another study done by Raza and Vaum found $68 \%$ sensitivity and $95 \%$ specificity with Doppler Ultrasonography. It also stated $85 \%$ and $88 \%$ positive and negative predictive values respectively. It has been shown in the past, that high resolution ultrasonography can detect 3 to 4 cancers per 1000 women in asymptomatic cases. ${ }^{24}$ Despite of this, biopsies are still generally required as a follow up or adjunct to any of the ultrasonography techniques that we currently have.

\section{Magnetic resonance imaging (MRI)}

This is another obliging screening tool which has been shown to reveal malignancies that are not seen on mammograms. It is especially useful in case of multifocal, multicentric or contralateral diseases. ${ }^{25}$ Density of breast tissue, presence of implants or scars can decrease the sensitivity and specificity of mammography and ultrasound. ${ }^{26}$ This fact makes it quite efficient in ruling out the diagnosis of breast cancer. ${ }^{27}$ It can help to delineate and differentiate benign lesions like the fibroadenomas, thus reducing the need for needless biopsies. Recent improvements to the MRI are in the areas of spatial resolution and temporal resolution which means that small cancers like carcinoma in situ can now be safely ruled out. Contrast-Enhanced MRI (CEMRI) has been shown to have sensitivity of more than $90 \%$, which 
gives it an advantage over previously described screening methods However, low specificity (of about $72 \%$ ) found in some research studies, gives it a setback. ${ }^{28}$ It is said that it has about $30 \%$ more false positives than mammography. Another drawback is of course the cost. MRI still remains an expensive screening tool. MRI is therefore not indicated for all patients as it is best reserved for patients with a relatively high risk of advanced breast cancer. ${ }^{29,30} \mathrm{~A}$ better use of MRI would be in pre-operative workup and staging of the disease. ${ }^{31}$

\section{$3 D$ (three-dimensional) imaging}

3D imaging techniques have literally added a new dimension in screening and diagnostic tools for breast cancer. 3D mammography and 3D MRI are the forerunners in this category. 3D mammography is sometimes referred to as breast tomosynthesis ${ }^{32}$ and it is becoming widely used in screening for breast malignancies as it is said to detect a slightly higher number of cancers than the conventional mammography. However, dilemma prevails about whether this technique actually provides benefit over 2D mammography or it over-diagnoses the indolent lesions. ${ }^{33}$ Three dimensional MRI is becoming a prevalent tool which offers an advantage in form of accurately detecting tumour extension. A better picture provided by 3D MRI can improve the efficacy of surgical treatment of breast cancer. ${ }^{34}$ However; the major limiting factor for these modalities is the very high cost, compared to other methods. $^{3}$

\section{Nuclear imaging}

This is one of the recent techniques and it is also regarded as molecular breast imaging. It makes use of a radioactive agent (tracer) which is injected into the vein and absorbed by body tissues including tissues in the breast. Cancerous cells generally absorb more of the radioactive tracer than normal cells and this is the case with cancerous breast cells as well. After the injection of the tracer, the cancer cells are imaged. There are two main techniques that are used here. ${ }^{35}$

- The breast-specific gamma imaging (BSGI): This uses gamma rays as its radioactive tracer and works by similar mechanism as described above. The sensitivity and specificity of BSGI are found to be $94.45 \%$ and $90.93 \%$ respectively. The major indications for BSGI include pre-operative workup, follow up after surgery, lesions which cannot be detected by mammography or ultrasonography, presence of multiple benign lesions and presence of suspicious lesions. ${ }^{36}$

- The positron emission tomography (PET): This uses sugars like F-fluorodeoxyglucose as the radiotracer that uses glucose metabolism pathway to detect cancer. ${ }^{37,18}$ Most of these radiotracers are consumed more by the tumour cells. PET is said to be of more help in recording post chemotherapy examinations or follow up when checking for recurrence. Lind and colleagues stated $96 \%$ and $77 \%$ sensitivity and specificity respectively. ${ }^{38}$ Its major setback is in it not been able to detect small cancers which make many question its use as a screening test.

In these techniques, the images are taken after the injection of a radioactive tracer which allows the cancer cells to be properly delineated. The drawback of nuclear imaging in breast cancer screening is the amount of radiation emitted to the patient which is almost 2-15 times more than that with conventional mammogram. This is accentuated by the fact that the tracer is given intravenously. So even though it is an effective screening tool, further researches are required to discover ways of reducing the radiation exposure in molecular breast imaging. ${ }^{39}$

\section{GENETIC SCREENING}

\section{BRCA screening}

This is not necessarily a cancer screening as it does not detect cancer. It, however, is a marker that detects the likelihood of a woman's chances of developing cancer. Such women, who show a high risk of developing cancer from BRCA screening, should then undergo more rigorous screening test like the MRI screening, for example. The mutations in BRCA-1 and BRCA-2 oncogene are the most commonly observed genetic defects associated with breast cancer. ${ }^{40,41}$ These BRCA genes can also influence therapy of breast cancer also. ${ }^{42,43}$ As per U. S. Preventive Services Task Force, BRCA screening should be offered to women with a strong family history of breast, ovarian, fallopian tube or peritoneal cancer and mutation of BRCA. ${ }^{44}$ High cost of BRCA testing makes it unavailable for universal accessibility. Further research is needed to analyse the false positive results indicating further testing and unnecessary preventive surgeries.

\section{Biopsies}

This is the confirmatory diagnostic test for malignancies, where breast tissue is taking ether through an open surgery or minimally invasive core-needle. Core-needle method offers less complications and shorter recovery time. As it is a diagnostic test, it is only indicated when there is strong evidence from screening test that the cancer is very much likely. ${ }^{45}$ The sensitivity of core needle biopsy ranges between 91-99\%, depending upon the simultaneous use of other guiding imaging technique. Similarly, the specificity ranged between $92-98 \% .^{46}$

\section{CONCLUSION}

Breast cancer screening remains a significant medical topic today because it carries with it life-saving potential when properly applied. Of course there are different methods available for breast cancer screening and 
improving technology means that current methods can be improved upon. To conclude the review, the initial screening methods still include mammography and ultrasonography. MRI and nuclear imaging can be reserved for preoperative workup or advanced stages of the disease. Genetic screening methods should be adopted for identifying the "at risk" women, for breast cancer. Biopsy still remains the gold standard test for confirming the diagnosis of breast cancer.

Funding: No funding sources

Conflict of interest: None declared

Ethical approval: Not required

\section{REFERENCES}

1. Chlebowski R. Nutrition and physical activity influence on breast cancer incidence and outcome. The Breast. 2013;22:S30-7.

2. Torre L, Bray F, Siegel R, Ferlay J, Lortet-Tieulent J, Jemal A. Global cancer statistics, 2012. CA: A Cancer J Clinicians. 2015;65(2):87-108.

3. Nover A, Jagtap S, Anjum W, Yegingil H, Shih W, Shih W et al. Modern Breast Cancer Detection: A Technological Review. Int J Biomed Imaging. 2009;2009:1-14.

4. Science Daily. Major differences between male and female breast cancers uncovered, but male patients still disadvantaged by lack of research. Science Daily. 2016. Available at: https://www.sciencedaily. com/releases/2016/03/160310080508.htm. Accessed on 11 September 2018.

5. World Health Organization. Early detection of cancer. Who.int. 2018. Available at: http://www. who.int/cancer/detection/. Accessed on 11 September 2018.

6. Hong Kong Cancer Registry. Overview of Hong Kong Cancer Statistics of 2015. Www3.ha.org.hk. 2018. Available at: http://www3.ha.org.hk/ cancereg/pdf/overview/Summary\%20of\%20CanStat \%202015.pdf. Accessed on 11 September 2018.

7. Mant D, Vessey M, Neil A, McPherson K, Jones L. Breast self examination and breast cancer stage at diagnosis. Br J Cancer. 1987;55(2):207-211.

8. U.S. Preventive Services: Task Force. www.uspreventiveservicestaskforce.org. 2016. Available at: https://www.uspreventiveservices taskforce.org/Page/Document/RecommendationStat ementFinal/breast-cancer-screening1\#Pod6.

Accessed on 11 September 2018.

9. Cancer Statistics Review, 1975-2011- Previous Version - SEER Cancer Statistics Review. Seer.cancer.gov. 2018. Available at: https://seer. cancer.gov/csr/1975_2011/. Accessed on 11 September 2018.

10. Narod S, Iqbal J, Giannakeas V, Sopik V, Sun P. Breast Cancer Mortality After a Diagnosis of Ductal Carcinoma In Situ. JAMA Oncol. 2015;1(7):888.

11. Colditz G, Rosner B, Chen W, Holmes M, Hankinson S. Risk Factors for Breast Cancer
According to Estrogen and Progesterone Receptor Status. JNCI J National Cancer Institute. 2004;96(3):218-28.

12. Hankinson S, Eliassen A. Circulating Sex Steroids and Breast Cancer Risk in Premenopausal Women. Hormones and Cancer. 2010;1(1):2-10.

13. Hahn K. Breast self-examination: defining a cohort still in need. Breast Diseases: A Year Book Quarterly. 2010;21(2):134-5.

14. Tarawneh E, Al-Atiyyat N. Exploration of Barriers to Breast-Self Examination and Awareness: A Review. Middle East J Nurs. 2013;7(6):3-7.

15. Kösters J, Gøtzsche P. Regular self-examination or clinical examination for early detection of breast cancer. Cochrane Database of Systematic Rev. 2003.

16. Saslow D, Hannan J, Osuch J, Alciati M, Baines C, Barton M, et al. Clinical Breast Examination: Practical Recommendations for Optimizing Performance and Reporting. CA: A Cancer J Clin. 2004;54(6):327-44.

17. Moss S, Wale C, Smith R, Evans A, Cuckle H, Duffy S. Effect of mammographic screening from age 40 years on breast cancer mortality in the UK Age trial at 17 years' follow-up: a randomised controlled trial. The Lancet Oncol. 2015;16(9):1123-32.

18. Tabar L, Yen M, Vitak B, Chen H, Smith R, Duffy S. Mammography service screening and mortality in breast cancer patients: 20-year follow-up before and after introduction of screening. The Lancet. 2003;361(9367):1405-10.

19. Chiarelli A, Edwards S, Prummel M, Muradali D, Majpruz V, Done S, et al. Digital Compared with Screen-Film Mammography: Performance Measures in Concurrent Cohorts within an Organized Breast Screening Program. Radiology. 2013;268(3):68493.

20. Miller A. The role of screening mammography in the era of modern breast cancer treatment. Climacteric. 2018;21(3):204-8.

21. Miller A, Wall C, Baines C, Sun P, To T, Narod S. Twenty five year follow-up for breast cancer incidence and mortality of the Canadian National Breast Screening Study: randomised screening trial. BMJ. 2014;348:g366-6.

22. Geisel J, Raghu M, Hooley R. The Role of Ultrasound in Breast Cancer Screening: The Case for and Against Ultrasound. Seminars in Ultrasound, CT and MRI. 2018;39(1):25-34.

23. Harvey J, Bovbjerg V. Quantitative Assessment of Mammographic Breast Density: Relationship with Breast Cancer Risk. Radiology. 2004;230(1):29-41.

24. Madjar H. Role of Breast Ultrasound for the Detection and Differentiation of Breast Lesions. BreastCare. 2010;5:109-14.

25. Hlawatsch A, Teifke A, Schmidt M, Thelen M. Preoperative Assessment of Breast Cancer: Sonography Versus MR Imaging. Am J Roentgenol. 2002;179(6):1493-501. 
26. Menezes G. Magnetic resonance imaging in breast cancer: A literature review and future perspectives. World J Clin Oncol. 2014;5(2):61.

27. Saslow D, Boetes C, Burke W, Harms S, Leach M, Lehman C, et al. American Cancer Society Guidelines for Breast Screening With MRI as an Adjunct to Mammography. Obstetrical Gynecological Survey. 2007;62(7):458-60.

28. Berg W, Gutierrez L, NessAiver M, Carter W, Bhargavan M, Lewis R, et al. Diagnostic Accuracy of Mammography, Clinical Examination, US, and MR Imaging in Preoperative Assessment of Breast Cancer. Radiology. 2004;233(3):830-49.

29. Hrung JM, Sonnad SS, Schwartz JS, Langlotz CP. Accuracy of MR imaging in the work-up of suspicious breast lesions: A diagnostic metaanalysis. Acad Radiol. 1999;6(7):387-37.

30. Morrow M. Magnetic Resonance Imaging in Breast Cancer. JAMA. 2004;292(22):2779.

31. Chapellier C, Balu-Maestro C, Bleuse A, Ettore F, Bruneton J. Ultrasonography of invasive lobular carcinoma of the breast: sonographic patterns and diagnostic value. Clin Imaging. 2000;24(6):333-6.

32. Bernardi D, Macaskill P, Pellegrini M, Valentini M, Fantò C, Ostillio L et al. Breast cancer screening with tomosynthesis (3D mammography) with acquired or synthetic 2D mammography compared with 2D mammography alone (STORM-2): a population-based prospective study. The Lancet Oncol. 2016;17(8):1105-13.

33. Houssami N, Lång $K$, Hofvind $S$, Zackrisson $S$, Bernardi D, Hunter $\mathrm{K}$ et al. Effectiveness of digital breast tomosynthesis (3D-mammography) in population breast cancer screening: a protocol for a collaborative individual participant data (IPD) metaanalysis. Translational Cancer Res. 2017;6(4):86977.

34. Nakamura S, Kenjo H, Nishio T, Kazama T, Doi O, Suzuki K. Efficacy of 3D-MR mammography for breast conserving surgery after neoadjuvant chemotherapy. Breast Cancer. 2002;9(1):15-9.

35. Hruska C. Molecular Breast Imaging for Screening in Dense Breasts: State of the Art and Future Directions. Am J Roentgenol. 2017;208(2):275-83.

36. Lee A, Chang J, Lim W, Kim B, Lee J, Cha E, et al. Effectiveness of Breast-Specific Gamma Imaging
(BSGI) for Breast Cancer in Korea: A Comparative Study. The Breast J. 2012;18(5):453-8.

37. Hankinson S, Eliassen A. Circulating Sex Steroids and Breast Cancer Risk in Premenopausal Women. Hormones and Cancer. 2010;1(1):2-10.

38. Hausegger K, Lind P, Reinprecht P, Igerc I, Beyer T. Advantages and limitations of FDG PET in the follow-up of breast cancer. Eur J Nuclear Med Molecular Imaging. 2004;31(0):S125-S134.

39. Hendrick R. Radiation Doses and Cancer Risks from Breast Imaging Studies. Radiology. 2010;257(1):246-253.

40. Force U. Genetic risk assessment and BRCA mutation testing for breast and ovarian cancer susceptibility: recommendation statement. 2005. Ann Intern Med. 2005;143(5):355-61.

41. Moyer V. Risk Assessment, Genetic Counseling, and Genetic Testing for BRCA-Related Cancer in Women: U.S. Preventive Services Task Force Recommendation Statement. Ann Internal Med. 2014;160(4):271-81.

42. Euhus D. Tamoxifen and Contralateral Breast Cancer in BRCA1 and BRCA2 Carriers: An Update. Breast Diseases: A Year Book Quarterly, 2007;17(4):401-2.

43. Nelson R. Tamoxifen Cuts Risk for Breast Cancer in BRCA Carriers. Medscape. 2013. Available at: http://www.medscape.com/viewarticle/809174 Accessed on 11 September 2018.

44. Moyer V. Risk Assessment, Genetic Counseling, and Genetic Testing for BRCA-Related Cancer in Women: U.S. Preventive Services Task Force Recommendation Statement. Ann Internal Med. 2014;160(4):271-81.

45. Agency for Healthcare Research and Quality. Having a breast biopsy. A review of the research for women and thier families. Available at: https://effectivehealthcare.ahrq.gov/sites/default/file s/pdf/breast-biopsy-update_consumer.pdf. Accessed on 11 September 2018.

46. Siegel R, Miller K, Jemal A. Cancer statistics, 2015. CA: A Cancer J Clin. 2015;65(1):5-29.

Cite this article as: Javaeed A. Breast cancer screening and diagnosis: a glance back and a look forward. Int J Community Med Public Health 2018;5:4997-5002. 